\title{
Assessing Expression of MUC1 and ZAG Protein Biomarkers in Prostate Biopsies Improves Prediction of Adverse Pathology Following Radical Prostatectomy
}

\author{
Nazish Durrani $^{1, \#}$, Mark Waldron ${ }^{2, \#}$, Catherine Cherry ${ }^{3,4, \#}$, Laurence Harewood ${ }^{5, \dagger}$, \\ Mark Frydenberg ${ }^{6, \dagger}$, John Pedersen ${ }^{7,8, \S}$, John Mills ${ }^{3,7,8, \S, a, *}$
}

${ }^{I}$ Department of Vascular Surgery, Sir Charles Gardiner Hospital, Nedlands, Perth, WA, Australia 6009

${ }^{2}$ School of Science and Technology, University of New England, Armadale, NSW, Australia 2350

${ }^{3}$ Department of Infectious Diseases, Monash University and The Alfred Hospital, and the Center for Biomedical Research, Burnet Institute, Melbourne, VIC, Australia 3004

${ }^{4}$ Faculty of Health Sciences, University of The Witwatersrand, South Africa

${ }^{5}$ University of Melbourne and Epworth Health Care Group, Richmond, VIC, Australia 3121

${ }^{6}$ Monash University and Australian Urology Associates, Malvern, VIC, Australia 3144

${ }^{7}$ TissuPath Specialist Pathology, Mount Waverley, VIC, Australia 3149

${ }^{8}$ Monash University Faculty of Medicine, Central Clinical School, Melbourne, VIC, Australia 3004

\begin{abstract}
Objectives: To determine whether assessing expression of MUC1 and ZAG proteins in prostate biopsies, by immunohistochemistry, improves prediction of radical prostatectomy histopathology, which in turn predicts longer-term outcomes.

Methods: We studied 231 consecutive patients managed by two experienced urologic surgeons (MF, LH). Each patient had prostate biopsies revealing cancer followed by a radical prostatectomy. Expression of MUC1 and ZAG in biopsy tissue was assessed by immunohistochemistry, masked to the radical prostatectomy histopathology. Data were analysed by Chi-square, Fischer exact test \& Mann Whitney U test followed by multivariate analysis using binary logistic regression.

Results: By univariate analysis, MUC1 expression in prostate biopsies was associated with worse histopathology in the radical prostatectomy specimen $(\mathrm{p}<0.023)$, while ZAG expression was associated with better pathology $(\mathrm{p}=0.03)$. By multivariate analysis decreased expression of ZAG in biopsies $(\mathrm{p}=0.02)$, but not MUC1 expression, improved prediction of high-risk radical prostatectomy pathology beyond conventional biopsy variables; neither MUC1 nor ZAG staining improved prediction of minimal-risk cancers.
\end{abstract}

Conclusions: Assessment of ZAG expression in prostate biopsies, and possibly MUC1 expression, may improve knowledge of prostate cancers in vivo or after radical prostatectomy.

Keywords: Biomarkers, MUC1, prostate biopsy, prostate cancer, ZAG.

\section{INTRODUCTION}

Prostate biopsies are well known to be poor predictors of the histopathology of prostate cancers in vivo, even when all preclinical data are considered (e.g. serum PSA concentration in conjunction with biopsy histopathology) [1-7].

\footnotetext{
*Address correspondence to this author at the Monash University \& Burnet Institute, 85 Commercial Road, Melbourne, Victoria, Australia 3004;

Tel: +61 419877 472; Fax: +6139804 8124;

E-mails: john.mills@monash.edu; john.mills@tissupath.com.au

$\#{ }_{\text {and }}^{\dagger}$ and ${ }^{\S}$-- equal contributors to this study

a -- lead and corresponding author
}

False-negative biopsies can occur because only a small proportion of the gland is sampled, and when cancer is found in biopsies, the biopsy Gleason scores frequently underestimate the Gleason scores after radical prostatectomy (RPx) histopathologic review $[1,3,8,9]$.

A number of studies have shown that immunohistochemical (IHC) assessment of prostate cancer expression of one or both of two proteins, MUC1 (a membrane-bound member of the mucin family of $\mathrm{O}$ glycosylated proteins) and ZAG (AZGP1, a zinc-binding alpha-2-glycoprotein) improves predictions of clinical outcomes beyond conventional variables [10-12]. However, 
these prior studies generally used IHC on tissue from RPx specimens rather than needle biopsy tissue.

The aim of this study was to determine whether assessment of MUC1 and ZAG expression in prostate needle biopsies would improve prediction of the histopathology of the RPx specimen, which in turn has been shown to predict longer-term outcomes [7, 13-20]. If assessing MUC1 and/or ZAG expression in biopsies improved prediction of RPx pathology, beyond conventional variables, it could be used to assist in defining management strategies for all patients with prostate cancer, including those managed by watchful waiting, active surveillance or radiotherapy - circumstances where the histopathology of the prostate tumor in vivo is unknown and may remain so.

\section{METHODS}

This study was approved by the Human Research and Ethics Committee (HREC) of The Alfred Hospital (Approval No. 213/12). The Alfred HREC is constituted according to guidelines established by the Australian National Health and Medical Research Council.

We retrospectively assessed consecutive patients who had prostate biopsies showing PCa and then had a RPX performed by either of two experienced urologic surgeons (LH, MF). For each patient, the histopathology of the biopsy cores was evaluated by two of the three experienced uropathologists at TissuPath Specialist Pathology. RPx tissues were evaluated by one uropathologist using previously-described methods for RPx specimens, including calculation of prostate volume and cancer volumes [21]. For biopsies we recorded the number of cores obtained, the percentage of cores with cancer, the maximum length of tumor in a single core and the total length of cancer in all cores. Because that latter variable is partly operator-specific (the more cores, the greater the likelihood that total length of cancer will increase) we computed a mean cancer length per core (=[total cancer length]/[number of cores]). For both biopsies and RPx specimens, in addition to Gleason score, TissuPath uropathologists routinely estimated the proportion of the cancer that was Gleason pattern 4 ("\% pattern 4"), ranging from zero (a Gleason $3+3$ tumor) to $100 \%$ (a
Gleason 4+4/5 tumor; there were no biopsies with primary pattern Gleason 5). For example, cancers with 5\% to $<50 \%$ pattern 4 would have had a Gleason score of $3+4$, while those with $50 \%$ to $\leq 100 \%$ pattern 4 would have had a Gleason score of $4+3$. The advantage of this metric is that it is a continuous variable which also emphasizes the importance of Gleason pattern 4 for prognosis [22].

Demographic, histopathologic and clinical data (specifically, the preoperative serum concentration of PSA, in $\mathrm{ng} / \mathrm{ml}$ ) were obtained from pathology requests or the urologic surgeons' medical records; we also modeled "PSA density" using the prostate volume (in cc) determined after RPX (=[serum PSA concentration/[prostate volume $]$ ), as estimates of prostate volume by ultrasound were seldom recorded.

We divided RPx specimens into three groups, "highrisk", "intermediate risk", and "minimal-risk" (also often termed "indolent"). RPx cancers were deemed "high-risk" using the criteria proposed by O'Brien et al. and others which defined "high risk" as a tumor having any one of the following features: $\geq 50 \%$ of Gleason pattern 4 or 5 , tumor volume $\geq 4$.0cc, presence of seminal vesicle invasion (SVI), extraprostatic extension of the tumor (EPE), positive surgical margins (PSM), or PSA $>20$ [1, 23, 24]. Each of these variables except tumor volume has been shown to be independently predictive of PSA failure after RPX [7], while tumor volume has been shown to be significant in our studies [21]. "Minimal-risk" (also termed "indolent") prostate cancer in the RPx specimen was also defined by the O'Brien et al., criteria [1], as organ-confined disease (no SVI, EPE or PSM) with Gleason score $\leq 6$ and tumor volume $\leq 0.5 \mathrm{cc}$; these criteria are equivalent to those proposed earlier by others [18, 25-27]. "Intermediate-risk" cancers were those not defined as high- or minimal-risk.

Four micron sections of prostate biopsies were immunostained for MUC1 and ZAG using the Leica Bondmax system (Leica Microsystems Pty Ltd, North Ryde, NSW, Australia) and a novel, 2-color IHC system developed by TissuPath Specialist Pathology that allowed assessment of both proteins in single sections (See Fig. 1). After antigen retrieval the sections were first stained with a primary goat
A

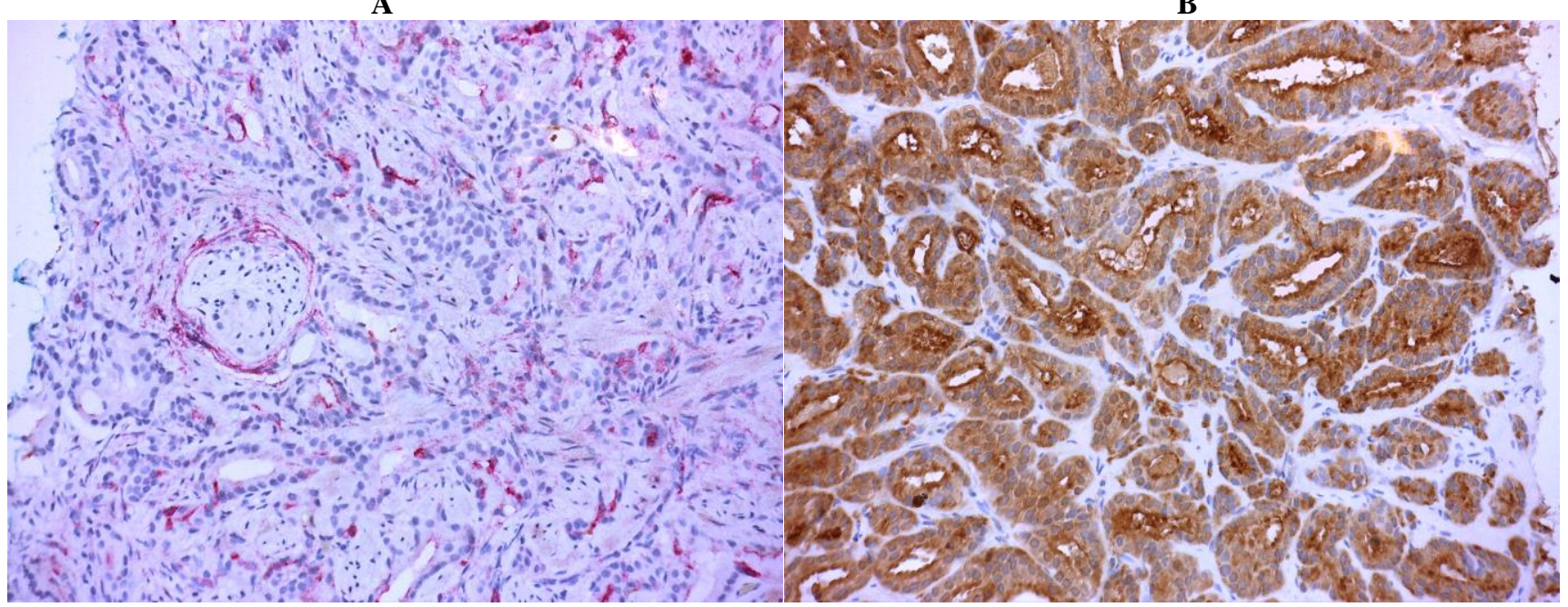

Fig. (1). Results of two-color immunohistochemical staining of tissues for MUC1 and ZAG. Photos at 400x magnification. Panel A: MUC1 staining only; Panel B: ZAG staining only. 
polyclonal IgG to ZAG (Santa Cruz Biotechnology catalog no. SC11238; ThermoFisher Scientific, Scoresby, VIC, Australia) followed by a mouse mab to MUC1 (Novacastra NCL-MUC1; Leica Microsystems Pty Ltd, North Ryde, NSW, Australia). The reaction was completed using Bond Polymer Refine Detection System (Leica). Tumor sections stained for MUC1 and ZAG expression were scored as either "no expression" (no staining) or "some expression" in which case the proportion of cancer cells staining was estimated ( $5 \%$ to $100 \%)$. The IHC data on the prostate biopsy sections were interpreted by a single expert uropathologist (JP) who was masked to the pathology of the RPx specimens. Only 13 $(5.1 \%)$ of the original 252 subjects had to be removed from analysis because of failure of the IHC reaction.

\section{Statistical Analysis}

Descriptive statistics were presented as median and interquartile range (IQR) for continuous variables, or percentages for categorical variables. Data that were not normally distributed were log-transformed; if that did not achieve normal distribution non-parametric statistics were used. Univariate analyses assessing pre-operative variables with RPx cancer risk type (high risk or minimal risk) were performed using t-tests, Wilcoxon rank sum tests or Chisquare tests as appropriate. Continuity corrections were used when $\mathrm{N}<5$. The results of Mann Whitney-U tests were reported as medians (interquartile range; IQR) and the results of the Chi-square analysis are expressed as percentage and count $(\mathrm{N})$ of the sample. Multivariate analysis of factors associated with cancer risk type was undertaken by logistic regression modelling using all factors associated on univariate analysis $(\mathrm{p}<0.1)$ along with any other factors associated with cancer risk type in other studies, followed by backwards elimination. Statistical significance was set at $\mathrm{p}<0.05$ (two-sided) for all analyses. Table 2 was created using IBM SPSS Statistics 20 (IBM Corporation, Armonk, New York, USA); all other analyses used Stata11 (StataCorp LP, College Station, Texas, USA).

\section{RESULTS}

Between October 2006 and March 2012 we ascertained 249 consecutive patients, managed by two experienced urologic surgeons (LH and MF), who had prostate biopsies followed by a RPx. A total of 231 subjects had all required data (Table 1) and were included in these analyses. The characteristics of this cohort were similar to other cohorts of patients treated by radical prostatectomy[1, 7, 28, 29]. The median number of ultrasound-guided, trans-rectal biopsies taken for each subject was 17 (IQR, 15-20) and apical cancer was present in 152 subjects (66\%). Over two-thirds of biopsies had Gleason scores of $3+3$ or $3+4$, with less than a third having primary Gleason pattern 4 (none had primary pattern 5).

Preoperative biopsies imperfectly predicted the histopathology of the radical specimen as been shown by many other studies $[1,3,8,9]$. Our review of upgraded RPx specimens with Gleason $3+3$ biopsies indicated that many had other characteristics suggesting that the RPx Gleason scores might be $>6$, including PSA concentrations above 10 and more than $30 \%$ of the biopsy cores showing cancer.

The proportion of cancer cells in biopsies expressing either MUC1 or ZAG proteins ("IHC staining") was essentially dichotomous, as a frequency histogram of percent staining for both proteins in all 231 subjects showed a break point nadir of $40 \%$ of cancer cells expressing the protein. Consequently we considered staining by either MUC1 or ZAG as positive if $>40 \%$ of cells expressed the protein, while $\leq 40 \%$ staining was considered as negative. ZAG expression was much more common than MUC1 expression, particularly if "expression" was restricted to samples with $\geqq 40 \%$ of cancer cells expressing ZAG protein (Table 1).

High-risk pathology (as defined in the Methods section) was found in well over half of the radical prostatectomy specimens (Table 1). All except one of the 16 subjects with SVI also had EPE, and that one subject had a $4.5 \mathrm{cc}$ tumor. Only two $(<2 \%)$ of the high-risk subjects were identified as such solely on the basis of PSA $>20$ [24]. Less than $14 \%$ of all RPx specimens had minimal-risk/indolent prostate cancers (as defined in the Methods section), although this was higher than the proportion seen in larger Australian (6\%, $5 \%)$ and German $(10 \%)$ cohorts [1, 25, 29]. Interestingly, 14 (29\%) of the 47 subjects with tumor volumes $\leq 0.5 \mathrm{cc}$ (some of whom had cancer volumes as small as $0.1 \mathrm{cc}$ ) had highrisk pathology, due to Gleason primary pattern 4 or 5 in all cases with concurrent EPE in one.

We assessed how well conventional pre-surgical variables, with the addition of MUC1 and ZAG expression, were associated with either high-risk (Table 2) or minimalrisk cancers (Table 3) by univariate analysis. High risk cancers generally had higher serum PSA concentrations, higher proportions of cores with cancer, higher median cancer length per core, and more adverse Gleason variables than not high-risk cancers (Table 2). MUC1 expression was more frequent and ZAG staining less frequent in the biopsies from high-risk cancers compared with those not high-risk, consistent with the known biology of these proteins in prostate cancer[10, 12, 30-33]. In those biopsies with $>40 \%$ MUC1 staining, the associated RPx specimens had a much higher percentage of pattern 4 histopathology than in those without biopsy MUC1 expression (median $70 \%$ versus $20 \%$, $\mathrm{p}<0.001$, Wilcoxon rank-sum test).

A univariate analysis of minimal risk disease $(\approx 14 \%$ of all RPx specimens) found only a few pre-operative variables differentiating them from riskier cancers, including lower PSA concentrations, less cancer seen in biopsies and less pattern 4 histology seen in biopsies (Table 3). Minimal risk disease at RPx was not associated with lower biopsy Gleason scores in this cohort $(\mathrm{p}=0.36)$. Although there were more ZAG-positive biopsies and fewer MUC1-positive biopsies in minimal-risk cancers compared with riskier cancers, neither MUC1 nor ZAG staining on biopsy was significantly associated with minimal risk prostate cancers (the proportion of minimal-risk cancers expressing ZAG or MUC1 was $15 \%$ and $11 \%$, respectively). 
Table 1. Characteristics of the entire study population $(\mathrm{N}=\mathbf{2 3 1})$.

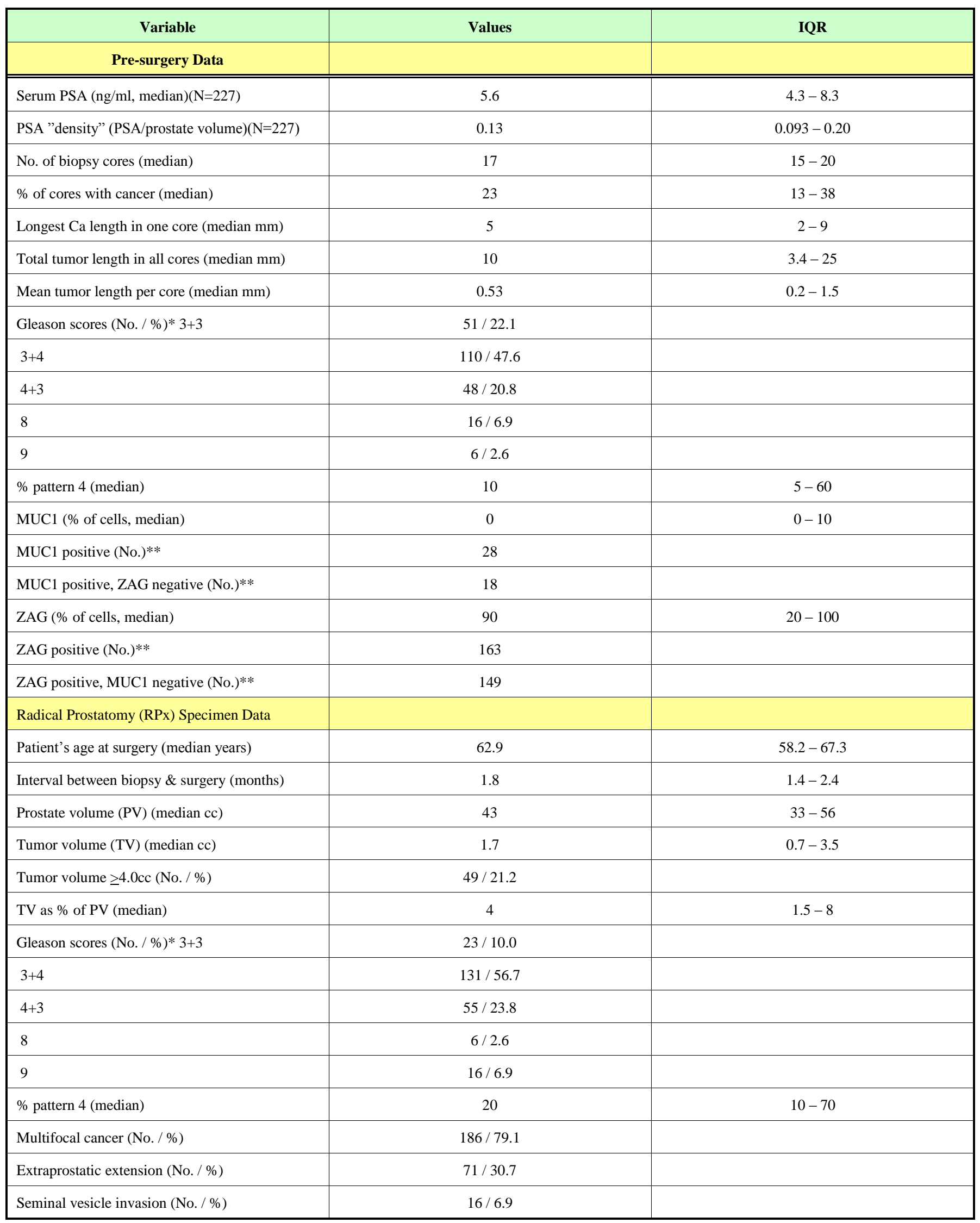


Table 1. contd.....

\begin{tabular}{|l|c|c|}
\hline \multicolumn{1}{|c|}{ Variable } & Values & IQR \\
\hline \multicolumn{1}{|c|}{ Pre-surgery Data } & & \\
\hline \hline Positive surgical margins (No. $/ \%)$ & $50 / 21.6$ & \\
\hline Hi-risk disease (No. $/ \%$ ) & $131 / 56.7$ & \\
\hline Minimal risk disease $($ No $/ \%)$ & $32 / 13.9$ & \\
\hline
\end{tabular}

*No cases of Gleason 10 or $1^{\circ}$ pattern 5. **IHC + or neg. as defined in Methods. Column heading abbreviations: IQR - interquartile ranges.

Table 2. Univariate analysis showing which pre-operative variables differentiate high-risk prostate cancers found at RPx from those not high risk.

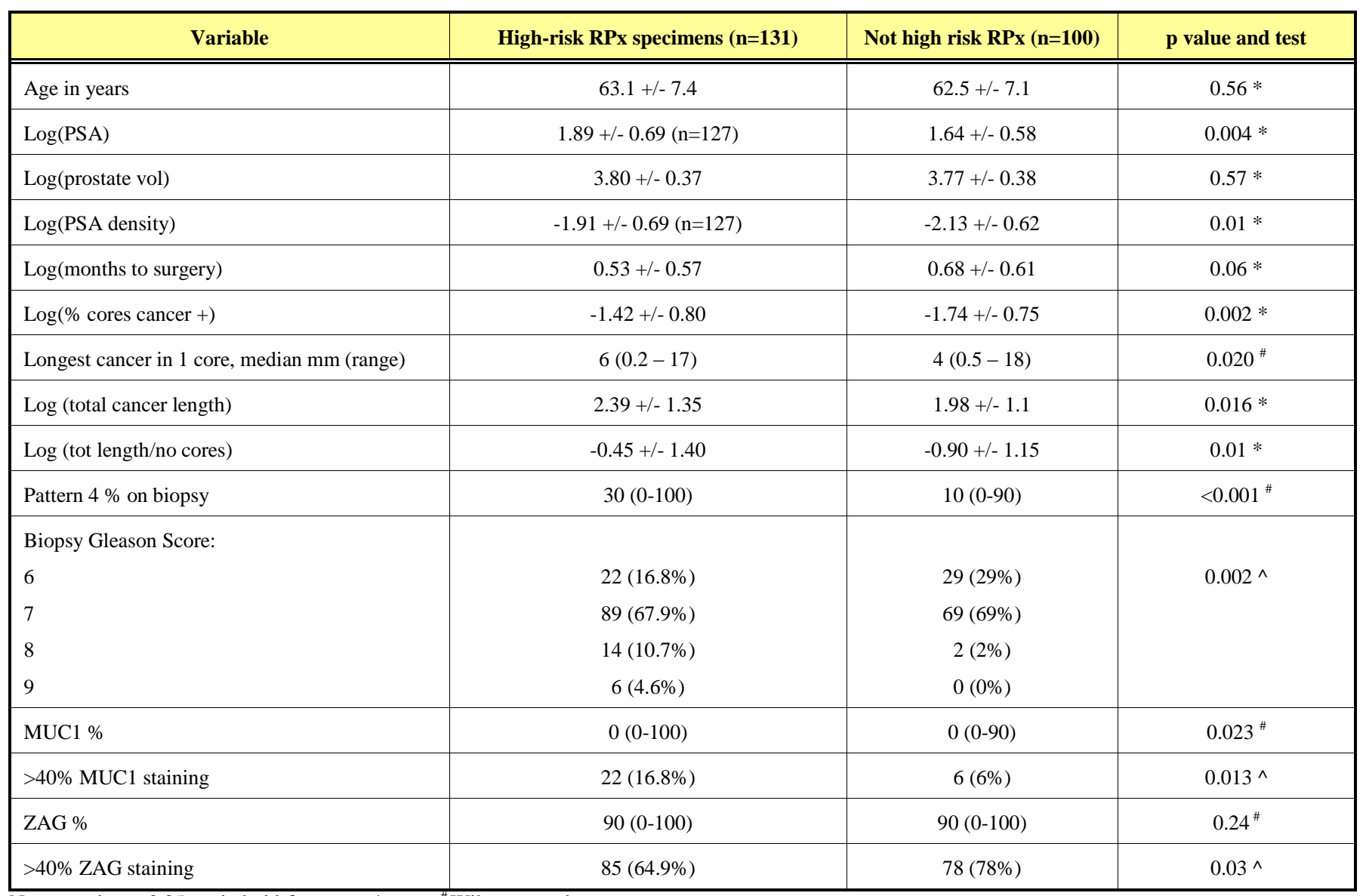

Note: $\mathrm{p}$ values $\leq 0.05$ are in bold-face type. ${ }^{*} \mathrm{t}$ test; ${ }^{\#}$ Wilcoxon rank sum test;

$\wedge$ Chi-square test

Logistic regression modeling to define independent variables determining high-risk cancers was undertaken by including all the conventionally available factors associated with high-risk disease from our univariate analysis $(\mathrm{p}<0.1)$ together with patient age (previously associated with highrisk disease in other studies (Tables 4a \& b) [34]. After a stepwise removal procedure, the conventional pre-surgical variables independently associated with high-risk prostate cancer were higher PSA, greater proportion of cores showing cancer and greater percentage of Gleason pattern 4 seen on biopsy (Table 4a). Consistent with previous reports [1], the predictive utility of these factors was only modest, with a model $\mathrm{R}^{2}$ of 0.13 . When modeling was repeated including
MUC1 and ZAG staining, having $>40 \%$ ZAG staining on biopsy was independently associated with a reduced likelihood of high-risk disease; consequently, inclusion of ZAG staining improved the ability to predict high-risk disease using pre-operative factors and increased model $\mathrm{R}^{2}$ by $15 \%$ to 0.15 (Table $4 \mathbf{b}$ ).

Multivariate analysis identified factors independently associated with minimum-risk cancers including lower PSA concentration, decreased percentage of cores with cancer and decreased percent of Gleason primary pattern 4 , as well as an unexpected association with increasing age (Table 5). The utility of conventional pre-operative factors for predicting minimal risk disease was modest, with model $\mathrm{R}^{2}=0.11$, and 
Table 3. Univariate analysis showing which pre-operative variables differentiate minimal risk disease found at $R P x$ from those without minimal risk disease.

\begin{tabular}{|c|c|c|c|}
\hline Variable & Minimum risk RPx $(\mathbf{n}=32)$ & NOT minimum risk RPx $(n=199)$ & $p$ value and test \\
\hline Age in years & $63.2+/-6.1$ & $62.8+/-7.4$ & $0.8 *$ \\
\hline $\log (\mathrm{PSA})(\mathrm{n}=195)$ & $1.50+/-0.70$ & $1.83+/-0.64$ & $0.009 *$ \\
\hline Log(prostate vol) & $3.74+/-0.43$ & $3.79+/-0.37$ & $0.48^{*}$ \\
\hline $\log ($ PSA density $)((n=195)$ & $-2.24+/-0.71$ & $-1.97+/-0.66$ & $0.03 *$ \\
\hline Log (months to surgery) & $0.68+/-0.49$ & $0.58+/-0.60$ & $0.4 *$ \\
\hline $\log (\%$ cores cancer +$)$ & $-1.85+/-0.78$ & $-1.52+/-0.78$ & $0.03 *$ \\
\hline Longest cancer in 1 core, median mm (range) & $4(0.5-12)$ & $5(0.2-18)$ & $0.05^{\#}$ \\
\hline Log (total cancer length) & $1.77+/-1.06$ & $2.28+/-1.27$ & $0.03 *$ \\
\hline Log (tot length/no cores) & $-1.10+/-1.10$ & $-0.57+/-1.33$ & $0.04 *$ \\
\hline Pattern $4 \%$ on biopsy & $5(0-70)$ & $20(0-100)$ & $<0.001^{\#}$ \\
\hline \multicolumn{4}{|l|}{ Biopsy Gleason Score: } \\
\hline 6 & $10(31.3 \%)$ & $41(20.6 \%)$ & $0.36^{\wedge}$ \\
\hline 7 & $21(65.6 \%)$ & $137(68.84 \%)$ & \\
\hline 8 & $1(3.1 \%)$ & $15(7.54 \%)$ & \\
\hline 9 & $0(0 \%)$ & $6(3.02 \%)$ & \\
\hline MUC1 \% & $0(0-90)$ & $0(0-100)$ & $1.0^{\#}$ \\
\hline$>40 \%$ MUC1 staining & $3(9.4 \%)$ & $25(12.6 \%)$ & $0.6^{\wedge}$ \\
\hline ZAG \% & $97.5(0-100)$ & $90(0-100)$ & $0.45^{\#}$ \\
\hline$>40 \%$ ZAG staining & $25(78.1 \%)$ & $138(69.4 \%)$ & $0.3^{\wedge}$ \\
\hline
\end{tabular}

* t test; \# Wilcoxon rank sum test; ${ }^{\wedge}$ ChI-square test

Table 4a. Results of logistic regression modeling to determine which conventional pre-operative factors are independently associated with high-risk prostate cancer, not including MUC1 or ZAG staining data.

\begin{tabular}{|c|c|c|c|}
\hline Factor & Odds Ratio & 95\% CI & p value \\
\hline \hline $\log (\mathrm{PSA})$ & 1.57 & $0.96-2.56$ & 0.07 \\
\hline $\log ($ cancer \%) & 1.31 & $0.88-1.96$ & 0.18 \\
\hline Percentage pattern 4 & 1.02 & $1.01-1.03$ & $<0.001$ \\
\hline
\end{tabular}

Overall model $\mathrm{p}<0.0001, \mathrm{R}^{2}=0.13$

Table 4b. Results of logistic regression modeling to determine which pre-operative factors are independently associated with high-risk cancer, including MUC1 \& ZAG staining.

\begin{tabular}{|c|c|c|c|}
\hline Factor & Odds Ratio & 95\% CI & p value \\
\hline \hline $\log ($ PSA $)$ & 1.60 & $0.97-2.64$ & 0.06 \\
\hline $\log ($ cancer \%) & 1.29 & $0.86-1.92$ & $<.22$ \\
\hline Percentage pattern 4 & 1.02 & $1.01-1.04$ & 0.001 \\
\hline$>40 \%$ ZAG staining & 0.47 & $0.25-0.90$ & 0.02 \\
\hline
\end{tabular}

Overall model $\mathrm{p}<0.0001, \mathrm{R}^{2}=0.15$.

inclusion of MUC1 and ZAG staining did not improve the model, with neither surviving the step-wise removal process.
Because data showing that MUC1 and ZAG expression have opposite predictive values of similar magnitude [10, 35], we substituted data from biopsies where only one 
Table 5. Results of logistic regression modeling to determine the conventional pre-operative factors associated with minimal-risk disease at prostatectomy.

\begin{tabular}{|c|c|c|c|}
\hline Factor & Odds Ratio & 95\% CI & p value \\
\hline \hline Age in years & 1.04 & $0.98-1.10$ & 0.16 \\
\hline $\log ($ PSA) & 0.49 & $0.25-0.94$ & 0.03 \\
\hline $\log ($ percentage cancer) & 0.69 & $0.39-1.21$ & 0.2 \\
\hline Percentage pattern 4 & 0.98 & $0.96-0.995$ & 0.02 \\
\hline
\end{tabular}

Overall model $\mathrm{p}=0.0003, \mathrm{R}^{2}=0.11$.

protein was expressed but the other was not (e.g. MUC1 $>40 \%$ but $\mathrm{ZAG} \leq 40 \%$ and vice versa - see Table 1 ) in our univariate and multivariate analyses. The results of these analyses were equivalent to those in Tables 3-5 (data not shown). We also looked at the pre-surgical variables predicting an RPx specimen with $>50 \%$ Gleason pattern 4 . The conventional factors predicting $>50 \%$ pattern 4 in the RPx specimen included serum PSA concentration, PSA density, proportion of cores with cancer, percent pattern 4 in the biopsy and Gleason score $>7$ in the biopsy, as well as MUC1 staining (\% pattern 4 on biopsy explained almost $35 \%$ of the variation). There was a trend towards reduced ZAG expression also predicting $>50 \%$ pattern 4 in the RPx specimen. The conventional pre-surgical variables produced a model with an $\mathrm{R}^{2}$ of 0.44 , while adding $\mathrm{MUC} 1$ increased the $\mathrm{R}^{2}$ only slightly to 0.45 .

We also assessed the utility of IHC expression of MUC1 and ZAG by constructing a receiver operating characteristic (ROC) curve. An ROC curve comparing the percentage of biopsy staining for MUC1 with the finding of a high-risk tumor after RPx showed the area under the curve (AUC) of 0.58 (95\% CI 0.51-0.64), confirming the at least modest predictive utility of assessing the expression of this protein. The ROC AUC for ZAG expression was 0.46 (CI 0.39-0.53).

We also analyzed 25 low-risk cancers and 90 high risk cancers with sole MUC1 or ZAG expression by IHC for their association with high-risk or minimal-risk cancers. Fourteen $(88 \%)$ of the 16 biopsies solely expressing MUC1 were associated with high-risk cancers, but only $16 \%$ of biopsies from these high-risk cancers expressing MUC1. Of 99 biopsies expressing solely ZAG, only $23 \%$ were biopsies associated with minimal risk cancers and $23(92 \%)$ of these biopsies were ZAG positive. However, $84 \%$ of biopsies from high-risk cancers also expressed ZAG.

\section{DISCUSSION}

This study is the first to assess the utility of MUC1 and ZAG expression in prostate biopsies for prediction of RPx histopathology in patients with prostate cancer. Adverse histopathology in the RPx specimen has been shown to be a surrogate for longer-term poor outcomes such as PSA failure and/or prostate cancer-related death [7, 17, 20, 25, 36-40]. On univariate analyses our data showed that MUC1 staining of prostate biopsies, compared with MUC1-negative biopsies, significantly predicted two adverse features of subsequent RPx specimens: a higher percent pattern 4 and a higher proportion of high-risk cancers. MUC1 staining also predominated in high-risk cancers compared with those that were low- or intermediate-risk. For ZAG staining, univariate analyses showed only a trend towards lower $\%$ pattern 4 and a lower proportion of high-risk cancers. By multivariate analysis, ZAG expression augmented conventional variables for prediction of high-risk cancers in that increased ZAG expression in biopsies decreased the likelihood of finding high-risk pathology in the RPx specimen. ZAG expression did not improve prediction of minimal-risk cancer by conventional variables, and MUC1 expression had little utility in the current cohort for prediction of either high- or minimal-risk pathology in RPx specimens.

Prior studies of RPx tissue have shown positive MUC1 or ZAG expression to be predictive of PSA failure and/or metastases, [10, 12, 30-33] but no studies have investigated these markers in prostate biopsies, although Gunia et al. showed that MUC1 expression in transurethral resection specimens ("incidental prostate cancers") predicted adverse pathology following subsequent radical prostatectomy [41]. The failure of our study to document a predictive capacity of MUC1 expression is most likely related to the small sample size (as relatively few biopsies stained MUC1 positive, the study lacked power to assess this biomarker), although it is also possible that its expression in biopsies is not strongly related to RPx pathology.

Overall this study supports adding IHC of biopsy tissue for expression of ZAG, and possibly MUC1, in subjects with localized cancer, to improve prediction of prostate cancer characteristics by current algorithms. IHC of course is ideal for translation into clinical practice, especially if it utilizes commercial antibodies, as it is available in almost all histopathology laboratories, even in the developing world, and it is already a procedure that is reimbursed in many jurisdictions. Because of the "molecular" heterogeneity of prostate cancers, IHC has the advantage of assessing protein expression throughout the tumor, in contrast to "molecular" methods which only sample a small part of any cancer [42]. In a separate, large cohort of prostate cancer patients, we have investigated a panel of protein biomarkers assessed by IHC, including MUC1 and ZAG; these data showed improved prediction of cancer-specific mortality beyond 'conventional' metrics with this protein panel, including MUC1 and ZAG [35]. These data support the results of the present study into MUC1 and ZAG staining by IHC.

Several recent studies have also attempted to improve prediction of RPx histopathology by proteomic or genomic assessments [43-45]. Although none of these proteomic studies focused on MUC1 or ZAG as potential candidates, it would be of interest to see whether adding these two 
proteins, validated in numerous other studies, to these more recent predictive algorithms would improve their predictive capacity.

\section{CONFLICTS OF INTEREST}

Prof Mills and A/Prof Pedersen are employees of TissuPath Specialist Pathology, but their compensation is not influenced in any way by these data or this publication. There are no other conflicts of interest.

\section{ACKNOWLEDGEMENTS}

This work was funded in part by a grant from TissuPath Research Pty Ltd. We acknowledge the support of our colleagues at TissuPath Specialist Pathology who contributed to this study: the expert pathologists who assessed the biopsies and RPx specimens incorporated in this study; and the talented laboratory staff at TissuPath, especially Alison Nanscawen and Jane Oxenford who developed and implemented the two-color IHC for MUC1 and ZAG essential for the success of this research. The authors affiliated with the Burnet Institute gratefully acknowledge the contribution to this work of the Victorian Operational Infrastructure Support Program received by the Burnet.

\section{REFERENCES}

[1] O'Brien BA, Cohen RJ, Ryan A, et al. A new preoperative nomogram to predict minimal prostate cancer: accuracy and error rates compared to other tools to select patients for active surveillance. J Urol 2011; 186: 1811-7.

[2] Parr RL, Mills J, Harbottle A, et al. Mitochondria, prostate cancer, and biopsy sampling error. Discov Med 2013; 15: 213-20.

[3] Epstein JI, Feng Z, Trock BJ, Pierorazio PM. Upgrading and downgrading of prostate cancer from biopsy to radical prostatectomy: incidence and predictive factors using the modified Gleason grading system and factoring in tertiary grades. Eur Urol 2012; 61: 1019-24.

[4] Bul M, van den Bergh RC, Rannikko A, et al. Predictors of unfavourable repeat biopsy results in men participating in a prospective active surveillance program. Eur Urol 2012; 61: 370-7.

[5] Bul M, Zhu X, Rannikko A, et al. Radical prostatectomy for lowrisk prostate cancer following initial active surveillance: results from a prospective observational study. Eur Urol 2012; 62: 195200.

[6] Hong SK, Sternberg IA, Keren Paz GE, et al. Definitive pathology at radical prostatectomy is commonly favorable in men following initial active surveillance. Eur Urol 2013; 66: 214-9.

[7] Walz J, Chun FK, Klein EA, et al. Nomogram predicting the probability of early recurrence after radical prostatectomy for prostate cancer. J Urol 2009; 181: 601-7.

[8] Corcoran NM, Casey RG, Hong MK, et al. The ability of prostatespecific antigen (PSA) density to predict an upgrade in Gleason score between initial prostate biopsy and prostatectomy diminishes with increasing tumour grade due to reduced PSA secretion per unit tumour volume. BJU Int 2011; 110: 36-42.

[9] Corcoran NM, Hovens CM, Hong MK, et al. Underestimation of Gleason score at prostate biopsy reflects sampling error in lower volume tumours. BJU Int 2012; 109: 660-4.

[10] Lapointe J, Li C, Higgins JP, et al. Gene expression profiling identifies clinically relevant subtypes of prostate cancer. Proc Natl Acad Sci USA 2004; 101: 811-6.

[11] Henshall SM, Horvath LG, Quinn DI, et al. Zinc-alpha2glycoprotein expression as a predictor of metastatic prostate cancer following radical prostatectomy. J Natl Cancer Inst 2006; 98: 14204.
[12] Yip PY, Kench JG, Rasiah KK, et al. Low AZGP1 expression predicts for recurrence in margin-positive, localized prostate cancer. Prostate 2011; 71: 1638-45.

[13] Han M, Partin AW, Pound CR, et al. Long-term biochemical disease-free and cancer-specific survival following anatomic radical retropubic prostatectomy: the 15-year Johns Hopkins experience. Urol Clin North Am 2001; 28: 555-65.

[14] Hoffman RM, Koyama T, Fan KH, et al. Mortality after radical prostatectomy or external beam radiotherapy for localized prostate cancer. J Natl Cancer Inst 2013; 105: 711-8.

[15] Hull GW, Rabbani F, Abbas F, et al. Cancer control with radical prostatectomy alone in 1,000 consecutive patients. J Urol 2002; 167: 528-34.

[16] Palisaar RJ, Graefen M, Karakiewicz PI, et al. Assessment of clinical and pathologic characteristics predisposing to disease recurrence following radical prostatectomy in men with pathologically organ-confined prostate cancer. Eur Urol 2002; 41: 155-61.

[17] Porter CR, Kodama K, Gibbons RP, et al. 25-year prostate cancer control and survival outcomes: a 40-year radical prostatectomy single institution series. J Urol 2006; 176: 569-74.

[18] Stamey TA, McNeal JE, Yemoto CM, et al. Biological determinants of cancer progression in men with prostate cancer. JAMA 1999; 281: 1395-400.

[19] Zincke H, Oesterling JE, Blute ML, et al. Long-term (15 years) results after radical prostatectomy for clinically localized (stage T2c or lower) prostate cancer. J Urol 1994; 152: 1850-7.

[20] Cooperberg MR, Hilton JF, Carroll PR. The CAPRA-S score: A straightforward tool for improved prediction of outcomes after radical prostatectomy. Cancer 2011; 117: 5039-46.

[21] Sherwin JC, Mirmilstein G, Pedersen J, et al. Tumor volume in radical prostatectomy specimens assessed by digital image analysis software correlates with other prognostic factors. J Urol 2010; 183: 1808-14.

[22] Stark JR, Perner S, Stampfer MJ, et al. Gleason score and lethal prostate cancer: does $3+4=4+3$ ? J Clin Oncol 2009; 27: 345964.

[23] Wilt TJ, Brawer MK, Jones KM, et al. Radical prostatectomy versus observation for localized prostate cancer. N Engl J Med 2012; 367: 203-13.

[24] D'Amico AV, Whittington R, Malkowicz SB, et al. Biochemical outcome after radical prostatectomy, external beam radiation therapy, or interstitial radiation therapy for clinically localized prostate cancer. JAMA 1998; 280: 969-74.

[25] Capitanio U, Ahyai S, Graefen M, et al. Assessment of biochemical recurrence rate in patients with pathologically confirmed insignificant prostate cancer. Urology 2008; 72: 1208-11.

[26] Epstein JI, Walsh PC, Carmichael M, Brendler CB. Pathologic and clinical findings to predict tumor extent of nonpalpable (stage T1c) prostate cancer. JAMA 1994; 271: 368-74.

[27] Steyerberg EW, Kattan MW, van der Kwast TH, et al. Prediction of indolent prostate cancer: validation and updating of a prognostic nomogram. J Urol 2007; 177: 107-12.

[28] Loeb S, Gonzalez CM, Roehl KA, et al. Pathological characteristics of prostate cancer detected through prostate specific antigen based screening. J Urol 2006; 175: 902-6.

[29] Samaratunga H, Delahunt B, Yaxley J, et al. Clinical significance of cancer in radical prostatectomy specimens: analysis from a contemporary series of 2900 men. Pathology 2014; 46: 11-4.

[30] Andren O, Fall K, Andersson SO, et al. MUC-1 gene is associated with prostate cancer death: a 20-year follow-up of a populationbased study in Sweden. Br J Cancer 2007; 97: 730-4.

[31] Cozzi PJ, Wang J, Delprado W, et al. MUC1, MUC2, MUC4, MUC5AC and MUC6 expression in the progression of prostate cancer. Clin Exp Metastasis 2005; 22: 565-73.

[32] Henshall SM, Quinn DI, Lee CS, et al. Altered expression of androgen receptor in the malignant epithelium and adjacent stroma is associated with early relapse in prostate cancer. Cancer Res 2001; 61: 423-7.

[33] Mills J, Oliver A, Sherwin JC, et al. Utility of RhoC and ZAG protein expression as biomarkers for prediction of PSA failure following radical prostatectomy for high grade prostate cancer. Pathology 2012; 44: 513-8.

[34] Siddiqui SA, Sengupta S, Slezak JM, et al. Impact of patient age at treatment on outcome following radical retropubic prostatectomy for prostate cancer. J Urol 2006; 175: 952-7. 
[35] Severi G, Fitzgerald LM, Muller DC, et al. A three-protein biomarker panel assessed in diagnostic tissue predicts death from prostate cancer for men with localized disease. Cancer Med 2014; 3: 1266-74.

[36] Shikanov S, Eggener SE. Hazard of prostate cancer specific mortality after radical prostatectomy. J Urol 2012; 187: 124-7.

[37] Corcoran NM, Hovens CM, Metcalfe C, et al. Positive surgical margins are a risk factor for significant biochemical recurrence only in intermediate-risk disease. BJU Int 2012: 110(6): 821-7.

[38] Eggener SE, Scardino PT, Walsh PC, et al. Predicting 15-year prostate cancer specific mortality after radical prostatectomy. J Urol 2011; 185: 869-75.

[39] Birkhahn M, Penson DF, Cai J, et al. Long-term outcome in patients with a Gleason score $\leq 6$ prostate cancer treated by radical prostatectomy. BJU Int 2011; 108: 660-4.

[40] Ahyai SA, Zacharias M, Isbarn H, et al. Prognostic significance of a positive surgical margin in pathologically organ-confined prostate cancer. BJU Int 2010; 106: 478-83.
[41] Gunia S, May M, Koch S, et al. MUC1 expression in incidental prostate cancer predicts staging and grading on the subsequent radical prostatectomy. Pathol Oncol Res 2010; 16: 371-5.

[42] Sboner A, Demichelis F, Calza S, et al. Molecular sampling of prostate cancer: a dilemma for predicting disease progression. BMC Med Genomics 2010; 3: 8.

[43] Shipitsin M, Small C, Choudhury S, et al. Identification of proteomic biomarkers predicting prostate cancer aggressiveness and lethality despite biopsy-sampling error. Br J Cancer 2014; 111: 1201-12.

[44] Lalonde E, Ishkanian AS, Sykes J, et al. Tumour genomic and microenvironmental heterogeneity for integrated prediction of 5year biochemical recurrence of prostate cancer: a retrospective cohort study. Lancet Oncol 2014; 15: 1521-32.

[45] Klein EA, Cooperberg MR, Magi-Galluzzi C, et al. A 17-gene Assay to predict prostate cancer aggressiveness in the context of gleason grade heterogeneity, tumor multifocality, and biopsy undersampling. Eur Urol 2014; 66(3): 550-60.

Received: November 17, 2014

Revised: January 19, 2015

Accepted: January 20, 2015

(C) Durrani et al.; Licensee Bentham Open.

This is an open access article licensed under the terms of the Creative Commons Attribution Non-Commercial License (http://creativecommons.org/licenses/ by-nc/3.0/) which permits unrestricted, non-commercial use, distribution and reproduction in any medium, provided the work is properly cited. 